\title{
Some Results on Formal Power Series and Differentiable Functions
}

\author{
By \\ Masahiro SHIOTA
}

\section{$\S$ 1. Introduction}

In [2] we see that any formal power series in two variables with coefficients in $\mathbf{R}$ or $\mathbf{C}$ (in this paper only the real case will be considered,) can be transformed to a polynomial by some automorphism change of the variables. In [3] Whitney shows an example which is a convergent series in three variables but which cannot be transformed to a polynomial. In this paper we give a formal power series example in three variables that is never transformed to be convergent ( $\S 2$ ).

A formal power series is the Taylor expansion of some $C^{\infty}$ function at the origin by $E$. Borel theorem. The followings refine it.

Theorem 1. Let $f$ be a formal power series in the variables $x=\left(x_{1}, \cdots, x_{n}\right)$. Let $K$ be a positive real. There exists a $C^{\infty}$ function $g$ defined on $|x|<K$ with the Taylor expansion at $0 \mathrm{Tg}=f$ and which is analytic except when $x=0$.

Theorem 2. There exists a homomorphism $S$ from the $\mathbf{R}$-algebra $\mathcal{F}$ of formal power series in one variable $x$ to the $\mathbf{R}$-algebra $\mathcal{E}$ of germs of $C^{\infty}$ function in one variable $x$ at 0 such that the composition $T \circ S$ is the identity homomorphism of $\mathscr{E}$.

There is a question in Malgrange [1] whether any homomorphism between the $\boldsymbol{R}$-algebras of $C^{\infty}$ function germs is a morphism (see $\S 4$ ). Theorem 2 gives a counter-example to it (Corollary).

Recieved March 20, 1975. 


\section{§ 2. An Example}

The example of Whitney is an analytic function $f$ in three variables of the form $x y(y-x)(y-(3+z) x)(y-\nu(z) x)$ where $\nu$ is a transcendental function with $\nu(0)=4$. If we replace the transcendental function above by a non-convergent formal power series, then $f$ cannot be transformed to a convergent one by any automorphism of the algebra of formal power series.

Proof. Suppose it is not so, then there exist formal power series in $(X, Y, Z)$-variables $x(X, Y, Z), y(X, Y, Z), z(X, Y, Z)$ such that $f(x(X, Y, Z), y(X, Y, Z), z(X, Y, Z))$ is analytic and that the determinant of Jacobian $D(x, y, z) / D(X, Y, Z)$ does not vanish at 0 . Moreover we can assume $\frac{D(x, y, z)}{D(X, Y, Z)}=\left(\begin{array}{lll}1 & 0 & 0 \\ 0 & 1 & 0 \\ 0 & 0 & 1\end{array}\right)$ at $(X, Y, Z)=(0,0,0)$. We know Zariski-Nagata Theorem and the fact that the formal power series ring and the convergent power series ring are unique factorization rings. Therefore there exist formal power series $g_{1}, \cdots, g_{5}$ in $(X, Y, Z)$-variables such that $g_{i}(0)=1$ for each $i, g_{1} \cdots g_{5}=1$, and $g_{1} \mathrm{x}$, $g_{2} y, \cdots, g_{5}(y-\nu(z) x)$ are convergent. Let $G_{i} i=1, \cdots, 5$ be $C^{\infty}$ functions in $(X, Y, Z)$-variables such that $T G_{i}=g_{i}$ and $G_{1} \cdots G_{5}=1$. We assume $g_{1} x, \cdots$ converge in a neighbourhood $U$ of $(X, Y, Z)=(0,0,0)$. Let $\phi_{1}=g_{1} x / G_{1}$, $\cdots, \phi_{5}=g_{5}(y-\nu(z) x) / G_{5}$ in $U$. Clearly $T \phi_{1}=x, \cdots, T \phi_{5}=y-\nu(z) x$, and the Taylor expansions of $\phi_{3}-\left(\phi_{2}-\phi_{1}\right), \phi_{4}-\left(\phi_{2}-(3+S) \phi_{1}\right)$ and $\phi_{5}-\left(\phi_{2}\right.$ $\left.-R(S) \phi_{1}\right)$ are zeros at 0 . Here $S, R$ are $C^{\infty}$ functions in $(X, Y, Z)-$, zvariables respectively such that $T S=z, T R=\nu$. From the assumption, $\left(g_{1} x, g_{2} y, Z\right)$ is an analytic local coordinates system around 0 . Hence $\phi_{1}^{-1}$ (0) $\cap \phi_{2}^{-1}(0)$ is an analytic curve. These imply that the functions $\phi_{3}-\left(\phi_{2}\right.$ $\left.-\phi_{1}\right), \cdots$ are zero identically on the curve $\phi_{1}^{-1}(0) \cap \phi_{2}{ }^{-1}(0)$. On the other hand $\left(\phi_{1}, \phi_{2}, Z\right)$ also is a local coordinates system around 0 . Thus, we find $C^{\infty}$ functions $\psi_{i j} i=3,4,5 j=1,2$ flat at 0 such that

$$
\begin{aligned}
& \phi_{3}-\left(\phi_{2}-\phi_{1}\right)=\psi_{31} \phi_{1}+\psi_{32} \phi_{2} ; \\
& \phi_{4}-\left(\phi_{2}-(3+S) \phi_{1}\right)=\psi_{41} \phi_{1}+\psi_{42} \phi_{2} ; \\
& \phi_{5}-\left(\phi_{2}-R(S) \phi_{1}\right)=\psi_{51} \phi_{1}+\psi_{52} \phi_{2} .
\end{aligned}
$$

Hence the intersection of any two $\phi_{i}^{-1}(0)$ are the same one $\phi_{1}^{-1}(0)$ 
$\cap \phi_{2}{ }^{-1}(0)$, and the intersection is described as $(X, Y, Z)=(X(Z), Y(Z), Z)$ where $X(Z)$ and $Y(Z)$ are analytic in $Z$-variable. We see easily that the Taylor expansions at 0 of the cross ratios of $\left(\phi_{1}^{-1}(0), \phi_{2}^{-1}(0), \phi_{3}^{-1}(0)\right.$, $\left.\phi_{4}^{-1}(0)\right)$ and $\left(\phi_{1}^{-1}(0), \phi_{2}^{-1}(0), \phi_{3}^{-1}(0), \phi_{5}^{-1}(0)\right)$ are $1 /(3+z(X(Z), Y(Z)$, $Z)$ ) and $1 / \nu(z(X(Z), Y(Z), Z))$, respectively.

In the same way we find analytic functions $\chi_{i j} i=3,4,5 j=1,2$ such that $\chi_{i j}(0) \neq 0$ and

$$
G_{i} \phi_{i}=\chi_{i 1} G_{1} \phi_{1}-\chi_{i 2} G_{2} \phi_{2} \quad i=3,4,5,
$$

and we see that the cross ratios above are $\chi_{31} \chi_{42} / \chi_{41} \chi_{32}$ and $\chi_{31} \chi_{52} / \chi_{51} \chi_{32}$, respectively. Hence they are analytic, but both $z(X(Z), Y(Z), Z)$ and $\nu(z(X(Z), Y(Z), Z))$ are not convergent by the assumption. That is a contradiction.

\section{$\S 3$. Proof of Theorem 1}

We prove only the case $n=K=1$. In the general case there is nothing to prove moreover.

Let $f$ be a formal power series $\sum a_{n} x^{n}$ where $a_{n}$ are reals. It is enough to find sufficiently large reals $m_{n}$ such that $\sum a_{n}(1-\exp (-1$ $\left.\left./ m_{n} x^{2}\right)\right) x^{n}$ converges on

(1) the real interval $[-1,1]$ with its each derivatives; and

(2) any compact subset of the complex domain $0<|x|<1$.

Proof of (1). For $n \geqq 2$ and $k \leqq n / 3$, we have

the $k$-th derivative of $\left(1-\exp \left(-1 / m x^{2}\right)\right) x^{n}$

$=n \cdots(n-k+1)\left(1-\exp \left(-1 / m x^{2}\right)\right) x^{n-k}+P(x, m) \exp \left(-1 / m x^{2}\right)$.

Here $P(x, m)$ is a polynomial in $x$ and uniformly converges to 0 when $m \rightarrow+\infty$. We can see that $\left(1-\exp \left(-1 / m x^{2}\right)\right) x^{n-k}$ and $\exp \left(-1 / m x^{2}\right)$ are monotonous in the intervals $[-1,0]$ and $[0,1]$ Hence these functions take the maximal values at $x=-1$ or 1 . Now, it follows that the $k$-th derivative of $\left(1-\exp \left(-1 / m x^{2}\right)\right) x^{n}$ uniformly converges to 0 when $m \rightarrow+\infty$ for $n \geqq 2$ and $k \leqq n / 3$. This proves (1).

Proof of (2) is also easy.

\section{§ 4. Homomorphism}

Proof of Theorem 2. Let $X$ be the ordered set consisting of the 
pairs $(A, \phi)$. Here $A$ is a subring of $\mathscr{F}$ containing $\mathbf{R}$ and $\phi$ is a homomorphism from $A$ to $\mathcal{E}$ such that the composition $T \circ \phi$ is the identity of $A$. Order two elements $(A, \phi),(B, \psi)$ of $X$ as follows

$$
(A, \phi) \leqq(B, \psi) \text { if } A \subset B \text { and }\left.\psi\right|_{A}=\phi .
$$

Apply Zorn's lemma, and $X$ has a maximal element $(A, \phi)$.

Now, we prove that $A$ of the maximal is itself $\mathscr{F}$. Assume that $A$ is a proper subset of $\mathscr{F}$, and that $\zeta$ is an element in $\mathscr{F}$ but not in $A$. There are two cases,

(1). $\zeta$ is algebraic over $A$;

(2). $\zeta$ is not so.

The case (1). Let $A[\zeta]$ and $A[t]$ be the ring generated by $\zeta$ over $A$ and the polynomial ring in $t$-variable with coefficients in $A$ respectively, and let $\theta$ be the homomorphism from $A[t]$ to $A[\zeta]$ naturally defined by $\theta(t)=\zeta$. Let $P(t)$ be an element of $\operatorname{ker} \theta$ whose degree as a $t$-polynomial takes the minimal in $\operatorname{ker} \theta$. For any element $Q$ of $\operatorname{ker} \theta$, dividing $Q$ by $P$ we have $Q Q^{\prime}=P P^{\prime}+R$ with $Q^{\prime} \in A, P^{\prime}, R \in A[t]$. Since $R \in \operatorname{ker} \theta$ and degree $R \leqq$ degree $P$, we see $R=0$. Hence we have the equality (a) $Q Q^{\prime}=P P^{\prime}$ for some $Q^{\prime} \in A-\{0\}$ and $P^{\prime} \in A[t]$. Let $P(t)=a_{1} t^{n}+\cdots$ $+a_{n+1}$. We may assume $\zeta=x^{s}$ for an integer $s$ through some change of the variable $x$. Let $\phi_{*} P(t)$ denote $\phi\left(a_{1}\right) t^{n}+\cdots+\phi\left(a_{n+1}\right)$. We shall define an extension homomorphism $\Phi$ of $\phi$ from $A[t]$ to $\mathcal{E}$ such that $T \circ \Phi(t)=x^{s}$ and $\Phi(\operatorname{ker} \theta)=0$. This follows from $(a)$ if we choose a germ $g(x)$ flat at 0 such that $\phi_{*} P\left(x^{s}+g\right)=0$. Let $y$ be a variable. Then $\phi_{*} P\left(x^{s}+y\right)$ is a polynomial $b_{1} y^{n}+\cdots+b_{n+1}$ in $y$ with coefficients in $\varepsilon$. We see that $b_{n+1}$ is flat at 0 and that $b_{n}=\left(\partial \phi_{*} P / \partial t\right)\left(x^{s}\right)$ is not flat. Because we have degree $(\partial p / \partial t)(t)=\operatorname{degree} P(t)-1$ and therefore $\partial p / \partial t \notin \operatorname{ker} \theta$. Put $y=x^{N} z$ for a sufficiently large $N$ and a new variable $z$. We can divide $b_{1} y^{n}+\cdots+b_{n+1}$ by $x^{N} b_{n}$. The quotient is $c_{1} z^{n}+\cdots+z$ $+c_{n+1}$, here $c_{i}$ are in $\mathcal{E}$ and $c_{n+1}$ is flat at 0 . Applying the implicit function theorem, we give a germ $z(x)$ flat at 0 such that $c_{1} z^{N}(x)+\cdots$ $+z(x)+c_{n+1} \equiv 0$. The germ $g(x)=x^{N} z(x)$ is what we want. Now we have defined a homomorphism $\Phi$. It is clear that $\Phi$ induces a homomorphism $\rho$ from $A[\zeta]$ to $\mathcal{E}$ such that the composition $T \circ \rho$ is the identity of $A[\zeta]$. This contradicts the maximality of $(A, \phi)$. Hence $A$ is $\mathscr{F}$. 
The proof of the case (2) is trivial from the proof of (1). Theorem 2 follows.

Remark. Even if we treat only the homomorphisms where the image of a convergent power series is naturally defined, there are infinitely many homomorphisms. We can prove this from the fact that any nonconvergent formal power series is algebraically independent over the convergent series ring.

Definition [1]. An endomorphism $u$ of $\mathcal{E}$ is called a morphism if there exists a germ $\phi$ with $\phi(0)=0$ such that for any $f \in \mathcal{E}$, we have $u(f)=f \circ \phi$.

The following answers the question in [1].

Corollary. The composed homomorphism $S \circ T$ is not a morphism. Here $S$ is defined in Theorem 2.

Proof. Suppose it is a morphism induced by some $\phi$. The first derivative of $\phi$ takes a non-zero value at 0 . Hence $S \circ T$ is an automorphism, on the other hand we have $S \circ T(f)=0$ for $f$ flat at 0 .

Remark. The general preparation theorem in [1] does not hold in the homomorphism case. That is, this $S \circ T$ is quasifinite but not finite.

\section{References}

L. 1] Malgrange, B., Ideals of differentiable functions, Oxford University Press, 1966.

[2] Shiota, M., On germs of differentiable functions in two variables, Publ. RIMS, Kyoto Univ., 9 (1974), 319-324.

[3] Whitney, H., Local properties of analytic varieties, Differential and Combinatorial Topology, Princeton Univ. Press, 1965.

Added in proof: The author was informed that Theorem 2 and its corollary were also obtained independently by K. Reichard (Manuscripta Math. 15 (1975), 243-250) and M. van der Put (to appear in Compositio Math.). 
